Data indicate high levels of trust and reliance upon clinicians' expert knowledge and accessibility of the service, flagging up potential tensions with self-management principles of valuing patient-held expertise, and fostering individuals' knowledge and skills to self-manage. Somewhat paradoxically, participant's geographical spread, combined with lack of specialists in local services, acts as both a driver to self-management, and towards reliance on specialist centres. Can increased reliance on specialist services be reconciled with self-management principles?

Twelve individuals were recruited from specialist clinics and participated in semi-structured interviews. Data were analysed against self-management principles informed by Social Cognitive Theory, and a tripartite theoretical conceptualisation of trust involving reliability, general, and specific trust. Data were then examined for incongruity and potential impact on implementation.

Service-users' trust in specialist services arises, firstly, from clinicians' understanding and knowledge of neuromuscular conditions, resulting in information perceived as good-quality and reliable; and secondly, from continuity and accessibility of care. This may naturally play towards health service-centred narratives, which are incompatible with self-management implementation. However, the data support alternative interpretations to align trust and reliance on specialist services with self-management. Opportunities for vicarious learning are sparse in rare conditions, but clinicians can promote self-efficacy through vicarious dialogue, and sharing experiences of other service-users. Trust acts as an enabler to goal-setting and positive risk-taking. Fundamentally, trust in the service may constitute a necessary pre-requisite without which a self-management dialogue is likely to be rejected.

Our preliminary findings highlight the uniqueness of patient-clinician relationships in this setting, providing insights into how the intervention can be implemented to this clinical context and population.

\section{THERAPEUTIC SETTINGS AND BEYOND: A TASK ANALYSIS OF RE-ESTABLISHING EPISTEMIC TRUST IN PSYCHOTHERAPY}

${ }^{1} \mathrm{E}$ Li, ${ }^{1} \mathrm{~N}$ Midgley, ${ }^{1} \mathrm{P}$ Luyten, ${ }^{2} \mathrm{C}$ Campbell. ' UCL; Anna Freud National Centre for Children and Families, UK; ${ }^{2}$ University College London, UK

\subsection{6/bmjopen-2021-QHRN.21}

Background The concept of epistemic trust captures one's willingness to receive new information as trustworthy and relevant, underpinning one's learning capacity to internalize new knowledge and generalize them in a wider context. The lack of epistemic trust may link to the emergence and persistence of general psychopathology. As a recently introduced concept in developmental and clinical psychology, epistemic trust requires empirical work never previously done.

Aims The aim is to explore how epistemic mistrust (e.g., hyper vigilance, petrification) come about in one's development leading to mental disturbance, how epistemic trust can be (re-)established in psychotherapy, and moreover, how therapists act can appropriately respond to epistemic mistrust and help establish epistemic trust.

Methods Task analysis, an inductive approach where patterns of change can be identified within a psychotherapy context, will be conducted in randomly selected audiorecordings of psychotherapy sessions from the Improving Mood with Psychoanalytic and Cognitive Behaviour Therapy (IMPACT) study, a randomized controlled trial comparing three interventions in the treatment of depression in adolescents.

Expected results A model of (re-)establishing epistemic trust in psychotherapy for depressed adolescents will be presented by engaging cases with both good and poor outcomes, with an awareness of the potentially different productive processes used across three treatment arms. The results answer whether levels of epistemic trust moderate one's psychotherapy outcomes and propensity to thrive in socially adverse circumstance-allow the extent to which epistemic trust underpins resilience to be further understood. Moving beyond specific mental disorders and psychotherapy orientations, we will argue how a lack of epistemic trust is common in general psychopathology and its restoration is necessary for all kinds of treatments based on our findings to help guide the professionals in practice.

The current stage of the work: Writing a study protocol.

\section{ENGAGING COMMUNITY: WAYS TO IMPROVE TRUST AND OUTCOMES THROUGH COMMUNITY-LED METHODS AND ANALYSIS}

K Luxion, M Svensson, D Frost. University College London, UK

\subsection{6/bmjopen-2021-QHRN.22}

Within the context of low-resource projects, both limited on time and resources, it can be difficult to incorporate equitable research methods, such as community-based participatory research. For LBGTQ+ research, there are similar barriers, with the need for health research projects that support community-led projects and centre LGBTQ+ voices through their methods. This presentation frames research methods as a social justice issue, addressing the value of community members as academic researchers; it seeks to highlight additional ways in which community involvement increases trust in both research processes and outcomes. The included examples are sub-projects and methods that are part of two distinct LGBTQ+/community-led PhD projects using either a multi-study or mixed methods approach; as such the discussion is focused on their use of qualitative methods. Coming from a social psychology perspective, themes are related to plurisexual emerging adults' parenthood desires and plans and a biomedical study on LGBTQ + pregnancy and parenthood, with recommendations that are transferable to other health topics. Focusing on the primary example, the presentation discusses the benefits of engaging LGBTQ+ community members assist on data processing and meaning-making while building qualitative research skills. A case study based on this postgraduateundergraduate mentoring scheme, proposes a purposeful approach to encouraging community-led research as part of capacity building efforts for undergraduates, within and beyond academia. Information to be covered, in addition to the case study, includes how participant trust was fostered through protocols, methods, and the analysis process - with further examples pending. 